\title{
Nanoscale topographical control of capillary assembly of nanoparticles
}

\author{
Valentin Flauraud', Massimo Mastrangeli ${ }^{2}{ }^{\dagger}$, Gabriel D. Bernasconi ${ }^{3}$, Jeremy Butet ${ }^{3}$, \\ Duncan T. L. Alexander ${ }^{4}$, Elmira Shahrabi ${ }^{11}$, Olivier J. F. Martin ${ }^{3}$ and Juergen Brugger ${ }^{5 \star}$
}

\begin{abstract}
Predetermined and selective placement of nanoparticles onto large-area substrates with nanometre-scale precision is essential to harness the unique properties of nanoparticle assemblies, in particular for functional optical and electrooptical nanodevices. Unfortunately, such high spatial organization is currently beyond the reach of top-down nanofabrication techniques alone. Here, we demonstrate that topographic features comprising lithographed funnelled traps and auxiliary sidewalls on a solid substrate can deterministically direct the capillary assembly of Au nanorods to attain simultaneous control of position, orientation and interparticle distance at the nanometre level. We report up to $100 \%$ assembly yield over centimetre-scale substrates. We achieve this by optimizing the three sequential stages of capillary nanoparticle assembly: insertion of nanorods into the traps, resilience against the receding suspension front and drying of the residual solvent. Finally, using electron energy-loss spectroscopy we characterize the spectral response and near-field properties of spatially programmable Au nanorod dimers, highlighting the opportunities for precise tunability of the plasmonic modes in larger assemblies.
\end{abstract}

T he design of nanoparticle clusters and their deterministic spatial arrangement are core opportunities ${ }^{1}$ as well as challenges $^{2}$ for nanotechnology. Nanofabrication by thin-film deposition, lithography and etching often produces nanostructures with grains and defects, which reduces their structural quality. In contrast, bottom-up assembly harnesses the superior and unique physico-chemical properties of semiconductor nanowires ${ }^{3}$, carbon nanotubes ${ }^{4}$, nanodiamonds ${ }^{5}$ and colloidal quantum dots $^{6}$, as well as metallic nanoparticles with tailored composition ${ }^{7}$, geometry ${ }^{8}$ and functionalization ${ }^{9,10}$. In particular, single-crystal and geometrically perfect metallic colloids are excellent building blocks for low-loss ${ }^{11}$ and strongly coupled plasmonic systems ${ }^{12}$. Fabrication approaches using DNA ${ }^{13}$ as both a linker and scaffold have demonstrated ångström-level accuracy in self-assembling clusters of molecules $^{14}$ and nanoparticles ${ }^{15-17}$. Yet building functional devices with a precise architecture additionally requires such a high degree of organization to be reconciled with a correspondingly accurate spatial placement of the nanocomponents over large areas ${ }^{18}$. Capillary assembly of nanoparticles ${ }^{19,20}$ may represent an effective technique for this purpose ${ }^{21}$. Capillary assembly achieves selective delivery of individual nanoparticles into suitable target sites when performed on substrates templated through topographical patterning and surface-chemical conditioning (Fig. 1) 22,23 . Thereby, capillary assembly of nanoparticles combines the complementary advantages of the chemical synthesis of nanoparticles ${ }^{2,15}$ with those of top-down nanofabrication, ideal for the precise definition of topographic features arbitrarily distributed over large surfaces.

Capillary assembly of colloids is attracting increasing attention ${ }^{24}$ and has witnessed several variations $s^{25-27}$ and extensions ${ }^{28,29}$. Yet, in spite of recent relevant insights ${ }^{30,31}$, complete understanding of the dynamics and the parametric dependence of its performance is still elusive. Here, we identify and investigate three distinct sequential stages in the dynamics of the capillary assembly of nanoparticlesnamely insertion, resilience and drying-that encompass the trajectory of single nanoparticles from the initial colloidal suspension to the final placement onto topographical substrates. We demonstrate that each stage significantly and distinctively affects the cumulative yield of the assembly process, and have designed effective traps geometrically tailored in all three dimensions to achieve an optimal assembly performance.

Sequential stages of capillary assembly of nanoparticles In the capillary assembly of nanoparticles, high assembly yield is correlated to dense nanoparticle packing in the accumulation zone (Fig. 1) ${ }^{22}$. Dense packing drastically quenches the Brownian motion of the nanoparticles, increasing the probability of nanoparticle sequestration into topographical traps. Deprived of thermal positional fluctuations, the nanoparticles in the accumulation zone are dragged across the underlying substrates exclusively by the receding motion of the triple contact line. For the Au nanorods used in this work (see Methods and Supplementary Fig. 1), anisotropic domains with smectic order extending over thousands of nanorods are visible from the surface of the accumulation zone (Supplementary Fig. 2a-e) ${ }^{30}$. Within the accumulation zone, multiple microscopic domains with short-range order are formed in a few nanorod monolayers adjacent to the substrate surface (Supplementary Fig. 2c,f). Each domain contains on average a few tens of uniformly aligned nanorods, and the domains span the entire range of orientations (Supplementary Fig. 3). The domains of nanorods in contact with the substrate prompt a new interpretation of prior experimental observations-specifically, the increase of assembly yield reported for an increased extension of the

\footnotetext{
${ }^{1}$ Microsystems Laboratory, Institute of Microtechnique, École Polytechnique Fédérale de Lausanne, 1015 Lausanne, Switzerland. ${ }^{2}$ Department of Bio, Electro and Mechanical Systems, Université Libre de Bruxelles, 1050 Bruxelles, Belgium. ${ }^{3}$ Nanophotonics and Metrology Laboratory, École Polytechnique Fédérale de Lausanne, 1015 Lausanne, Switzerland. ${ }^{4}$ Interdisciplinary Center for Electron Microscopy, École Polytechnique Fédérale de Lausanne, 1015 Lausanne, Switzerland. ${ }^{5}$ Microsystems Laboratory, Institute of Microtechnique and Institute of Materials, École Polytechnique Fédérale de Lausanne, 1015 Lausanne, Switzerland; †Present addresses: Physical Intelligence Department, Max Planck Institute for Intelligent Systems, 70569 Stuttgart, Germany (M.M.); Microelectronic Systems Laboratory, École Polytechnique Fédérale de Lausanne, 1015 Lausanne, Switzerland (E.S.). *e-mail: juergen.brugger@epfl.ch
} 


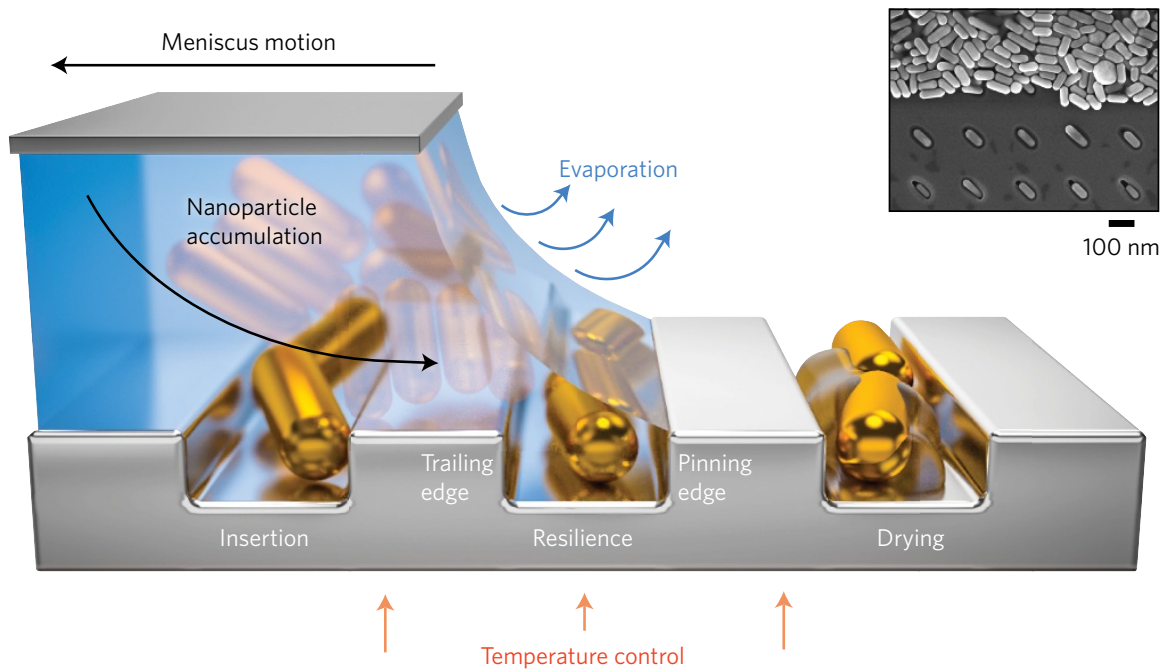

Figure 1 | Schematic of the capillary assembly of nanoparticles onto topographical traps of a low-wetting substrate (dimensions not to scale). A controlled volume of colloidal solution is confined ${ }^{24}$ between a patterned, low-wetting target substrate and a top plate set in relative sliding motion ${ }^{23}$. The colloidal suspension pins at the edge of the plate and is pulled across the templated substrate. Solvent evaporation from the receding solvent/vapour interface, which can be accelerated by substrate heating, induces a convective flow that drags nanoparticles from the bulk to the surface of the suspension ${ }^{51}$. The flow produces a dense accumulation of nanoparticles in the wedge-shaped region adjacent to the receding contact line (Supplementary Fig. 2a,b) and sustains the accumulation against nanoparticle back diffusion ${ }^{23}$. Steady-state nanoparticle accumulation is a function of the properties of the colloidal solution, surface tension, surfactant concentration and interparticle interactions ${ }^{38}$. The receding contact line drags the accumulation zone across the substrate, and the nanoparticles exit the colloidal meniscus to selectively fill shape-matching recessed traps, as shown in the inset. The three sequential stages of capillary assembly are illustrated from left to right: nanoparticles are successfully assembled if they (1) are inserted into a trap, (2) are not removed from the trap by capillary doctor blading or by friction with the sliding accumulation zone and (3) withstand displacement and reorientation within the trap during the final solvent drying.

accumulation zone $e^{32}$, and the independence of assembly yield on the relative orientation of nanotraps and receding contact line for a sufficiently extended accumulation zone ${ }^{30}$. Our hypothesis is that only a sufficiently extended accumulation zone can host enough domains of random orientation in its bottom layers, so that at least one of the domains that crosses a single trap of the substrate can tightly match the orientation of that very trap.

An entropy-driven phase transition ${ }^{33}$ orders the thin hard nanorods within the accumulation zone $\mathrm{e}^{30}$. The nanorods closest to the receding contact line orient their main axis parallel to the contact line across several adjacent rows (Supplementary Fig. 2b). These extremal nanorods may bias the filling of narrow aligned traps. However, under such tight confinement, loss of translational entropy ${ }^{33}$ excludes that nanorods rotate to fit into an underlying trap not parallel to the contact line. The exclusion of nanorod rotation is consistent with the domain-based trap-filling mechanism and curtails the validity of the commonly assumed mechanism ${ }^{34}$. The latter mechanism relies exclusively on the downward bending of the colloidal meniscus induced by contact line pinning ${ }^{34}$. Pinning of the receding contact line along solid edges ${ }^{35}$ transiently lowers the contact angle. Consequently, the local rate of solvent evaporation increases ${ }^{36}$ and the vertical component of the solvent/ vapour interfacial tension pushes the nanoparticles closest to the contact line onto the recessed traps.

Our experimental observations reveal that such description is incomplete and groups together a sequence of three distinct stages that characterize the filling of each trap. These stages (Fig. 1) need to be clearly differentiated to maximize assembly yield and positional accuracy. In the first stage, a nanoparticle is inserted into an unoccupied trap. According to our working hypothesis, the inserted particle belonged to the accumulation zone, yet it did not necessarily reside at the very edge of the meniscus. This was recently observed for spherical microparticles by real-time confocal imaging ${ }^{31}$. However, insertion per se does not ensure ultimate occupation of a trap. In the second stage, the inserted particle must withstand a possible removal. Nanoparticle removal from a trap may be triggered by, in sequence, the shearing action from the densely packed accumulation zone sliding across the substrate surface, and the eventual transient pinning of the receding contact line on the trapped nanoparticle itself. During sliding of the accumulation zone, an inserted particle may be removed and replaced ${ }^{31}$. Third, after insertion and resilience, a site-filling nanoparticle may be displaced by the evaporation of the residual solvent. The capillary immersion forces of the drying solvent affect the final relative pose of the nanoparticle(s) within topographical traps ${ }^{26,37}$.

\section{Enhancing traps through geometry}

In the following, we systematically investigate how single-crystal $\mathrm{Au}$ nanorods of a nominal size of $110 \mathrm{~nm} \times 40 \mathrm{~nm} \times 40 \mathrm{~nm}$ (Supplementary Fig. 1) are assembled by capillary means into large arrays of low-wetting traps nanomachined in rigid substrates to host single nanorods (see Methods and Supplementary Fig. 11). The capillary assembly of nanoparticles process was run simultaneously over all trap geometries described hereafter: straightedged, funnelled, straight-edged with auxiliary sidewall, and funnelled with auxiliary sidewall. The impact of the traps' geometrical features on the alignment precision and assembly yield of the nanorods-defined as the ratio of the number of traps filled with at least one nanorod and the total number of traps-can be interpreted through the sequence of insertion, resilience and drying stages of the nanorods.

We first consider the straight-edged trap (Fig. 2a,b and Supplementary Fig. 12), where the cross-section is constant across the entire depth ${ }^{22}$. Trap widening favours insertion, as evidenced by Fig. 2c. A low assembly yield of $15 \%$ at best was obtained for traps narrower than $50 \mathrm{~nm}$. This can be explained by the effective interaction diameter of the nanorods in suspension, which extends beyond the nominal diameter $(40 \mathrm{~nm})$ as a result of adsorbed surfactant, electric double layers and other boundary effects $^{38}$. Independently of trap width, trap depth improves the 


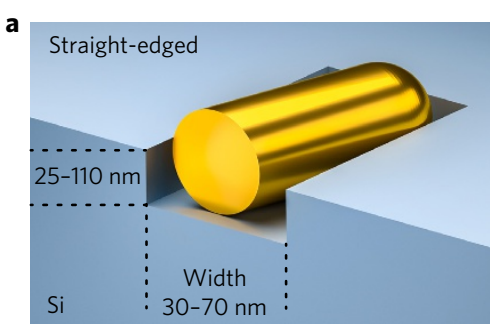

d
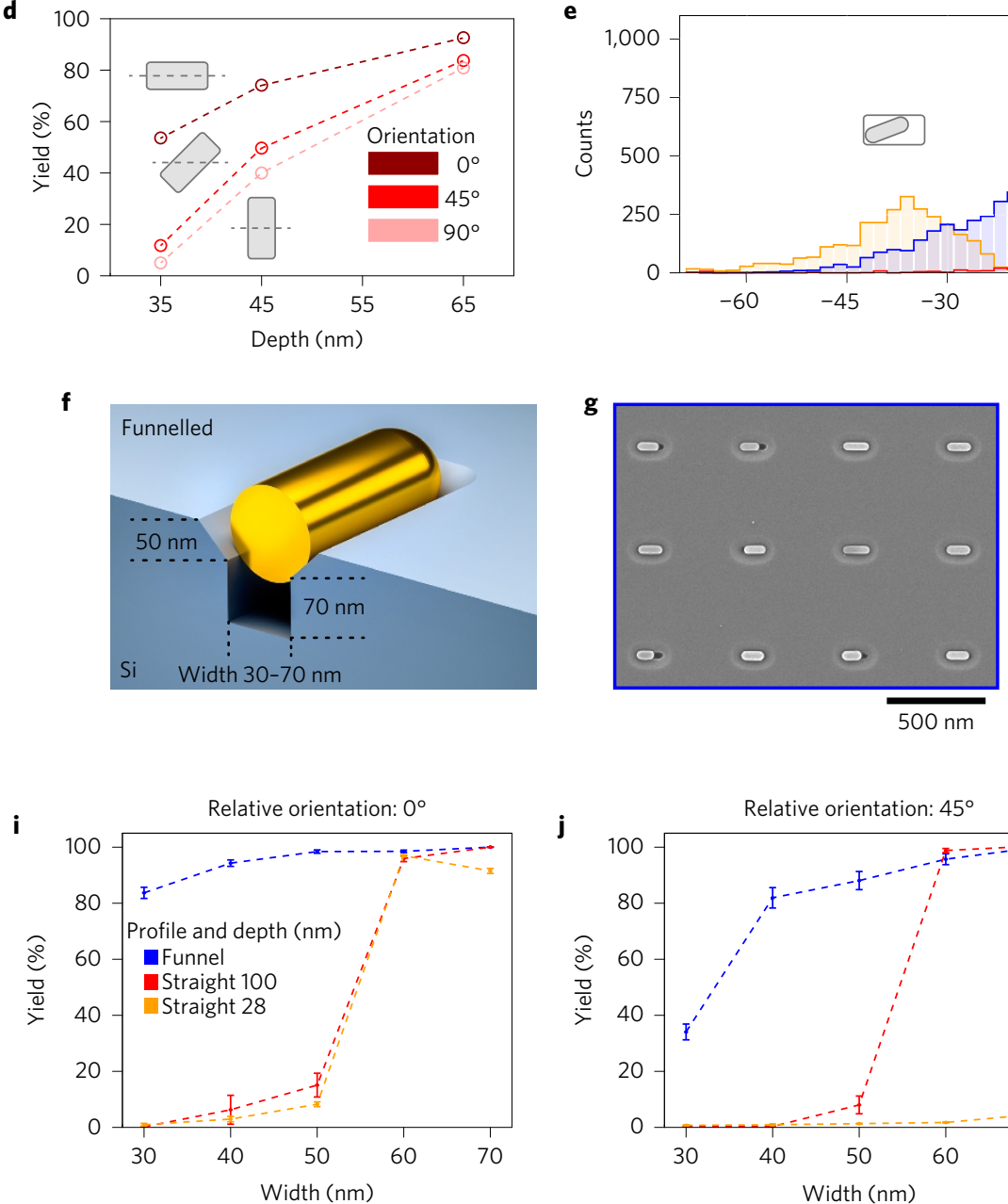

b

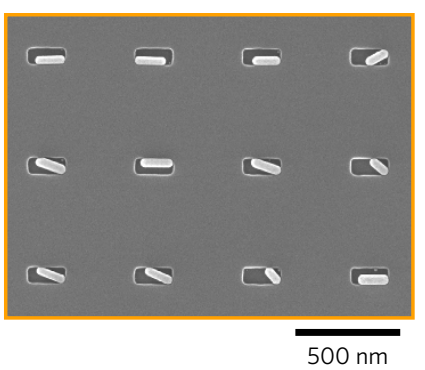

j

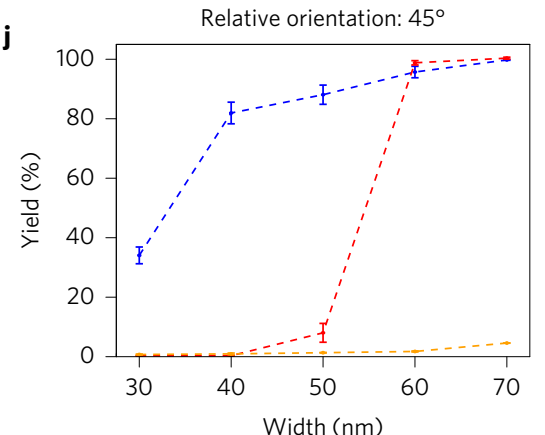

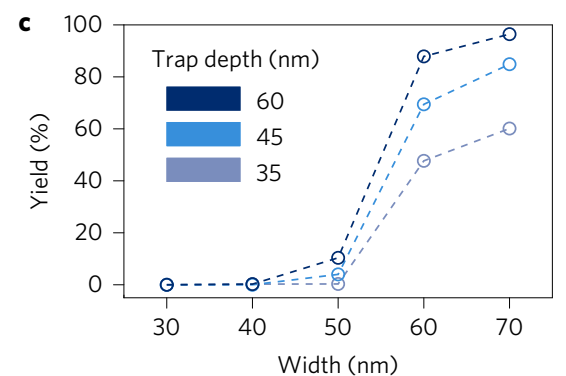

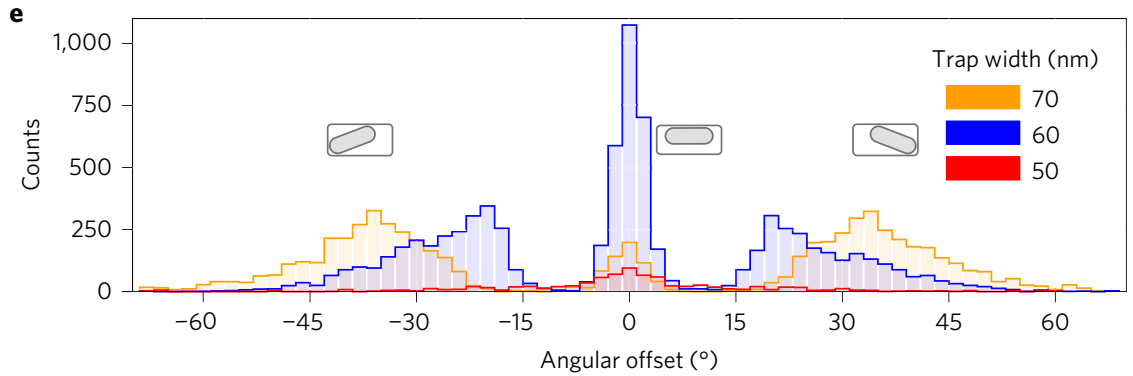

h

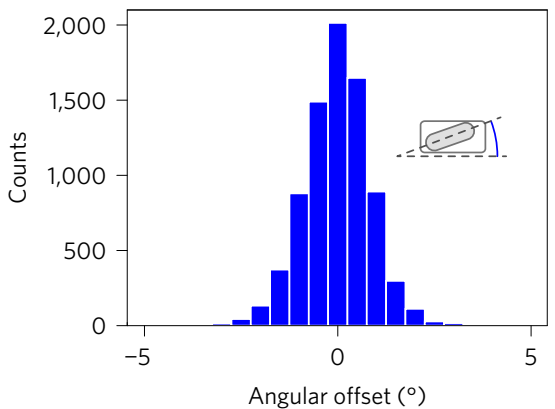

k

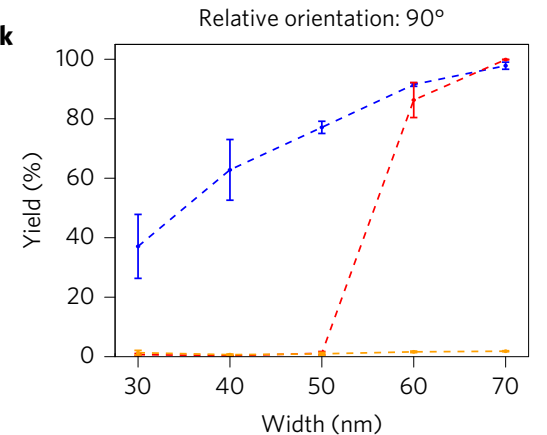

Figure 2 | Comparison of capillary assembly of Au nanorods in straight-edged and funnelled traps. a, A schematic representation of an Au nanorod assembled in a straight-edged trap. b, Electron micrograph of nominally $110 \mathrm{~nm} \times 40 \mathrm{~nm} \times 40 \mathrm{~nm}$ Au nanorods assembled in an array of 28-nm-deep straight-edged traps. c, Typical assembly yield versus trap width for straight-edged traps of depth smaller (35 nm), commensurate (45 nm) and larger $(60 \mathrm{~nm})$ than the nanorods' nominal diameter and with their main axis parallel to the receding contact line. $\mathbf{d}$, Dependence of assembly yield on trap depth and trap orientation relative to the receding contact line for 60-nm-wide traps. e, Trimodal distribution of assembled nanorods relative to the trap's main axis for 28-nm-deep traps. Local details of solvent drying in the low-wetting traps cause the adhesion to the closest topographical feature of misaligned nanorods (see insets). The angular dispersion of the off-axis nanorods reflects their length polydispersity. $\mathbf{f}$, A schematic representation of an Au nanorod assembled in a funnelled trap. g,h, Electron micrograph $(\mathbf{g})$ and angular distribution of nominally $110 \mathrm{~nm} \times 40 \mathrm{~nm} \times 40 \mathrm{~nm}$ Au nanorods assembled in an array of funnelled traps with 30-nm-wide bottom trench; the interquartile range is $1.07^{\circ}(\mathbf{h})$. i-k, Comparison of assembly yield versus trap width for shallow ( $28 \mathrm{~nm}$ ) and deep $(100 \mathrm{~nm})$ straight-edged traps and for the funnelled trap (error bars indicate 2 standard deviations around the mean value for each data point). More than one nanorod is assembled in the deep straight-edged traps, while this does not occur in funnelled traps with the same total depth.

assembly yield by providing a higher barrier against removal (Fig. 2c,d). For shallow traps of equal in-plane cross-section, the dependence of assembly yield on trap orientation with respect to the contact line (Fig. 2d) can also be attributed to the resistance against nanorod removal. Considering the removal mechanics of an inserted nanorod pivoting on the rear trap sidewall, nanorods lying in traps parallel to the receding contact line experience larger shearing and capillary traction, but their corresponding lever arm is also much smaller than that of nanorods resting in traps perpendicular to the contact line. This results in a smaller torque and thus a higher resistance against removal. Moreover, sufficiently deep traps are rather insensitive to the orientation with respect to the contact line (Fig. 2d). Capillary immersion forces between particles and sidewalls ${ }^{26,37}$ induce a trimodal angular 

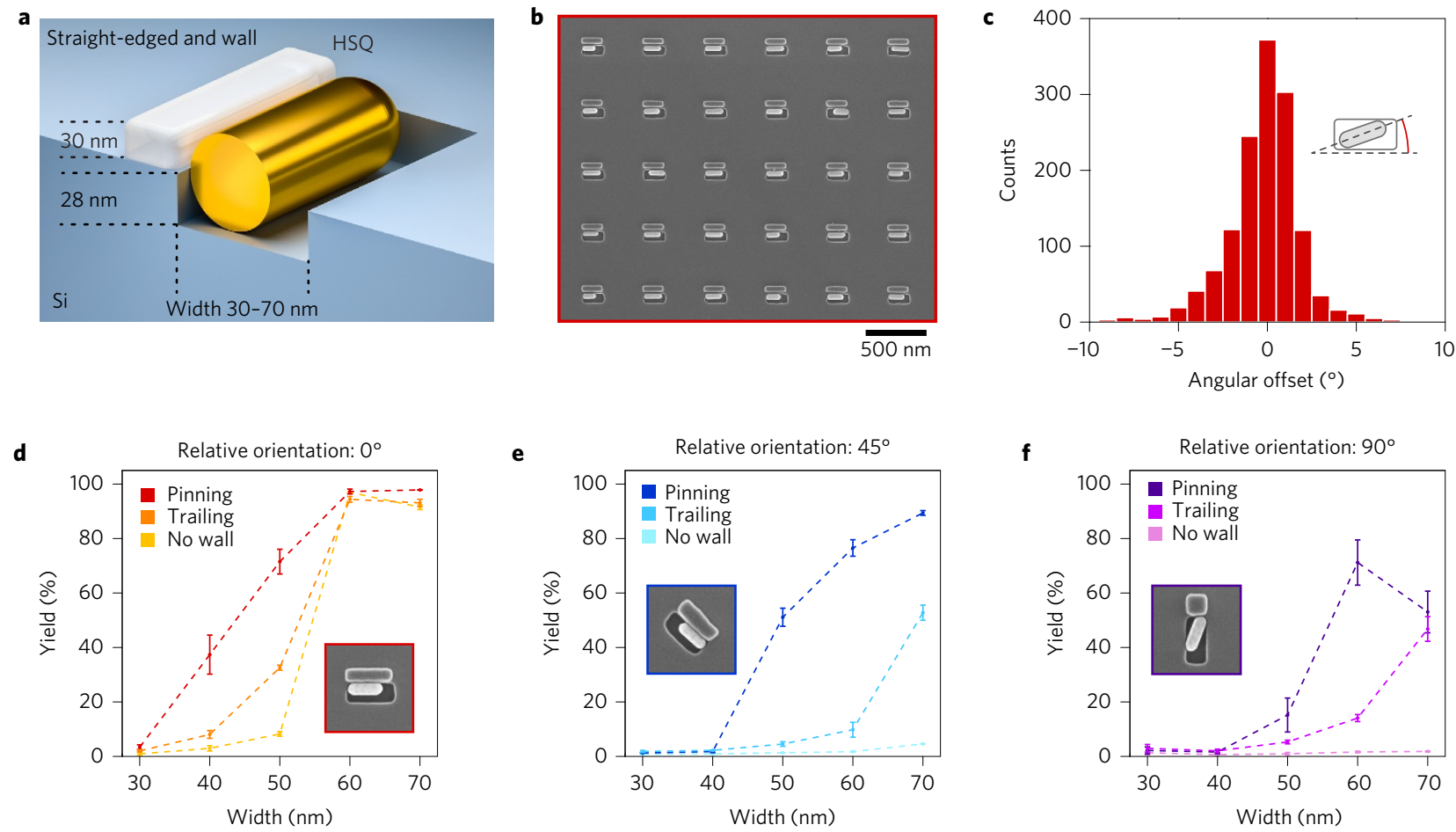

Figure 3 | Geometry and parametric assembly yield performance of the straight-edged trap with single auxiliary sidewall. a, Schematic representation of an Au nanorod assembled in a trap with straight-edged cross-section decorated by a single auxiliary HSQ sidewall. b, Electron micrograph of nominally $110 \mathrm{~nm} \times 40 \mathrm{~nm} \times 40 \mathrm{~nm}$ Au nanorods assembled in an array of traps of this type. c, Histogram of angular distribution of single nanorods assembled in 60-nm-wide traps featuring an HSQ sidewall on the pinning edge of the trap. $\mathbf{d - f}$, Comparison of assembly yield versus trap width for equal-sized traps of varying orientation and position of the auxiliary sidewall. Insets show the assembly of single nanorods in the respective traps. Error bars indicate 2 standard deviations around the mean value for each data point.

distribution for the nanorods assembled in our traps (Fig. 2b,e). More generally, nanorods assembled into straight-edged traps of width tightly accommodating the effective nanorod diameter in solution end up only loosely confined after drying, because of their reduced interaction diameter in the dry state. This inherent issue limits the placement accuracy achieved to date by using straightedged traps. On the other hand, sidewall-biased solvent evaporation can be exploited for predictable post-insertion orientation of nanorods within traps, as discussed below.

Hence, we designed and nanofabricated a three-dimensional trap geometry whereby a recessed funnel decorates the upper section of a straight-edged trap (Fig. 2f,g, and Supplementary Figs 4 and 13). The addition of the funnel enlarges the effective acceptance area for sliding nanorods and thus significantly increases the particle capture probability; moreover, the funnel precisely sequesters the inserted nanorod within a vertically tapered well. A bottom trench narrower than the effective particle diameter mechanically locks the nanorod during solvent evaporation precisely at the centre of the trap. This results in the nanorod being more tightly confined than in a straight-edged trap and counteracts nanoparticle removal by lowering its standing height (the comparison between trench-less funnelled and straight-edged traps of equal depth is provided in the Supplementary Information and Supplementary Fig. 5). The advantages of these features are reflected by the remarkable trapping performance. Assembly yield enhanced from 15\% without the funnel to $98 \%$ in the case of 50 -nm-wide funnelled traps parallel to the contact line, and from a negligible value it reached $83 \%$ in the case of 30-nm-wide funnelled traps. This is accompanied by extremely precise axial placement and particle orientation. Figure $2 \mathrm{~h}$ illustrates the small dispersion (interquartile range of $1.07^{\circ}$ ) in the angular distribution of single nanorods achieved in the 30-nm-wide funnelled traps. Funnelled traps show improved single-nanorod assembly yield compared with straight-edged traps for all tested trap widths and for all orientations of the traps relative to the receding contact line (Fig. 2i-k). The narrowest funnelled traps (30- and 40-nm-wide) provide ultimate positioning of the nanorods (Fig. 2g,h). Yet their absolute assembly yield is suboptimal (Fig. 2i-k) and shows that these traps are still prone to nanoparticle removal when they lie non-parallel to the receding contact line.

To further enhance the assembly performance through a more effective counteraction of nanoparticle removal, we placed an additional vertical barrier along a single edge of the traps. We specifically studied the effect of an auxiliary $30-\mathrm{nm}$-thick hydrogen silsesquioxane (HSQ) sidewall lithographically defined within $10 \mathrm{~nm}$ of a trap edge. When applied to a 28-nm-deep straight-edged trap (Fig. 3a,b, and Supplementary Figs 6a and 14), the auxiliary sidewall breaks the in-plane symmetry of the trap and biases the post-insertion solvent evaporation from the recessed trap. The spatial bias extends to capillary immersion forces, which consequently promote the displacement of the nanorod and its predictable adhesion to the decorated sidewall of the trap. These effects significantly improve accuracy and repeatability of nanorods' alignment. A very narrow monomodal angular distribution (interquartile range of $2.04^{\circ}$ ) is measured even for the widest cross-sections (Fig. 3c). The results in this case clearly evidence a superior assembly performance for traps with auxiliary sidewall on their pinning side (Fig. 3d-f).

The highest assembly yield was obtained with funnelled traps featuring the auxiliary sidewall either on the first (pinning) or second (trailing) trap edge crossed by the receding contact line (Fig. $4 \mathrm{a}-\mathrm{c}$ and Supplementary Fig. 15). In the former case, the unpinning of the receding contact line starts from a higher position, hence capillary gripping of an inserted nanoparticle is significantly inhibited. In the latter, the vertical barrier opposing the removal of the nanoparticle is elongated. The funnelled trap with auxiliary sidewall 

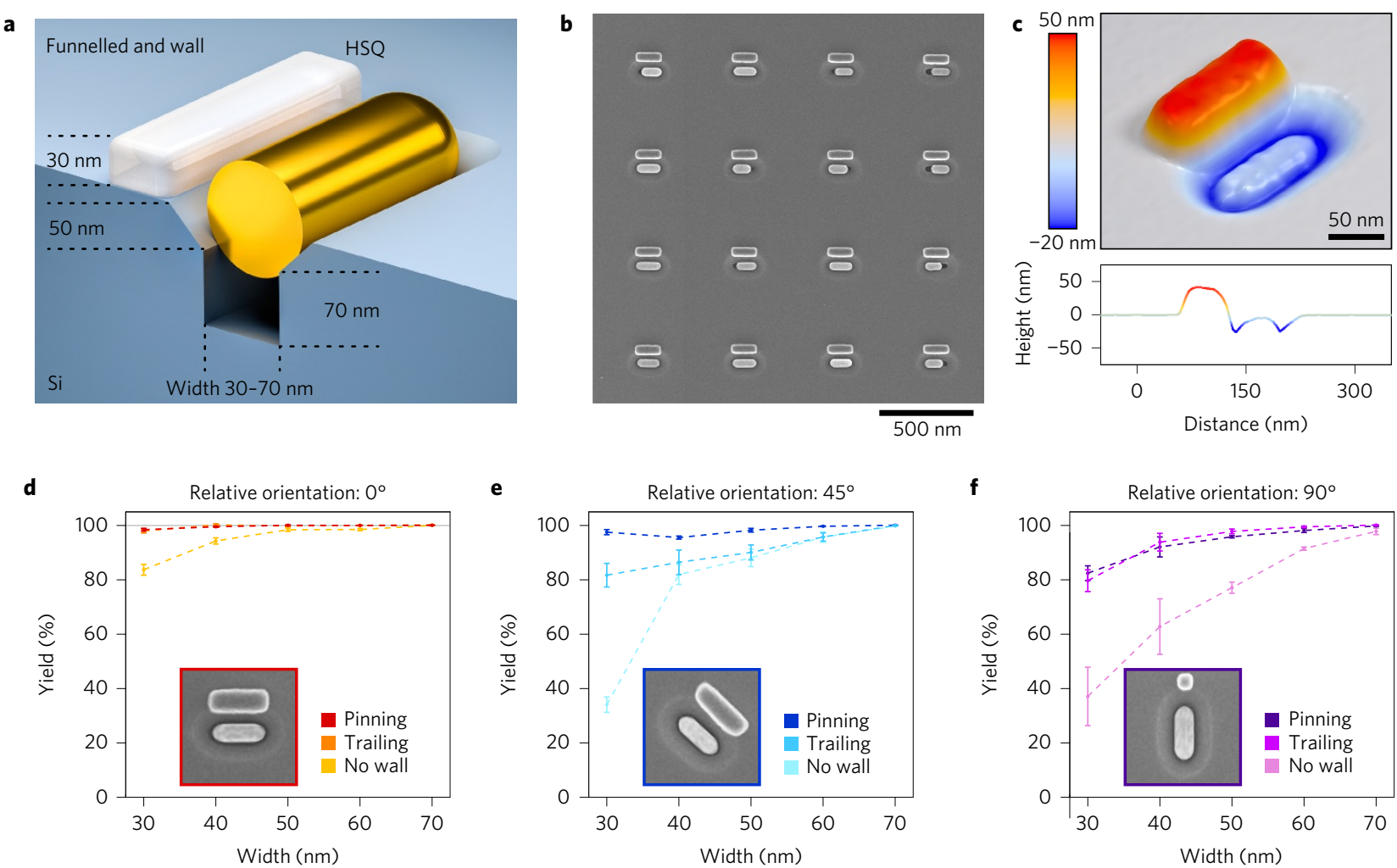

Figure 4 | Geometry and parametric assembly yield performance of the funnelled trap with single auxiliary sidewall. a, Schematic representation of an Au nanorod assembled in a trap with a funnelled cross-section and auxiliary sidewall. b. Electron micrograph of nominally $110 \mathrm{~nm} \times 40 \mathrm{~nm} \times 40 \mathrm{~nm}$ Au nanorods assembled in an array of traps of this type. c, Atomic force microscope image and corresponding linescan of a single Au nanorod assembled inside a funnelled trap decorated by an HSQ auxiliary sidewall. $\mathbf{d}-\mathbf{f}$, Comparison of assembly yield versus trap width measured for 4,800 equal-sized funnelled traps of each indicated type for varying trap orientation and position of the auxiliary sidewall. Insets show the assembly of single nanorods in the respective traps. Error bars indicate 2 standard deviations around the mean value for each data point.

delivers the best performance, as it efficiently exploits the mechanics of all the distinct stages of the capillary assembly of nanoparticlesnamely, nanoparticle insertion into the trap, resilience against removal, and final positioning by biased evaporation-to provide up to $100 \%$ assembly yield and positional control for all relative trap orientations (Fig. $4 \mathrm{~d}-\mathrm{f}$ ). Moreover, this performance is unaffected by overcrowding, since the single auxiliary sidewall allows excess nanoparticles to be dragged away from the trap by the sliding colloidal meniscus.

The predictable positional effects of the single auxiliary barrier can additionally be tailored to deterministically impose the longitudinal placement of nanorods along the main trap axis. This is demonstrated by single nanorods assembling within pairs of identical traps separated by a middle spacer, whose width can be lithographically predefined down to $5 \mathrm{~nm}$ according to needs (Supplementary Fig. 6b,c). Spatial proximity induces capillary coupling between adjacent traps, so that biased solvent evaporation makes the nanorods systematically adhere to the interposing spacer (Fig. 5a). Such deterministic positional control allows for the engineering of traps for spatially programmable arrangement of nanorod dimers with inner controllable nanogaps, an important feature of plasmonic systems ${ }^{12}$. Our approach combines nanometrelevel gap tuning through the length of surfactant adsorbed on the nanorods with the flexibility and larger spatial range achievable through lithographic methods over centimetre-scale areas.

\section{STEM-EELS characterization}

Nanorod dimers separated by preset gaps are prototypical examples of the opportunities opened by our insights into capillary assembly of nanoparticles for the fabrication of plasmonic structures (Fig. 5a).
The optical antennas are effectively composed of single-crystal arms, free of lossy adhesion layers ${ }^{39}$ and offer a large accessible surface $^{40}$. The smallest interparticle separation obtained in our assembled dimers measures less than $2 \mathrm{~nm}$ when mediated by collapsed surfactant layers, whereas a controlled oxygen plasma treatment enables the fusion of the facing nanorods into single structures connected by a narrow bridge (Fig. 5b). While nanometric interparticle gaps provide ultimate enhancement of the optical near-field ${ }^{41}$ along with appropriate platforms for the investigation of nonlocal effects ${ }^{42,43}$, these structures also offer extremely limited optical volumes and low stability against postprocessing $^{44,45}$. Metal-enhanced fluorescence is a typical example where optimal performance is obtained at interparticle distances of multiple nanometres where near-field enhancement and nonradiative decay processes occurring in the vicinity of the metal surface must be balanced ${ }^{46}$. Using electron energy-loss spectroscopy in a scanning transmission electron microscope (STEM-EELS) we demonstrate the effective tunability of our plasmonic dimers where interparticle distance is varied to specifically engineer large modifications in resonance wavelengths, near-field intensities and mode volumes to suit various target applications. We specifically studied the plasmonic response of dimer nanoantennas assembled onto thin silicon nitride membranes (see Methods), as presented in Fig. 5c-f. The trap geometry with straight edges and single auxiliary sidewall is best suited for the TEM measurements since it minimizes the substrate thickness under the nanorod dimers, thus enabling high-resolution imaging and spectral measurements with high signal-to-noise ratio. When compared with other near-field techniques such as scanning near-field optical microscopy, STEM-EELS is ideal for characterizing our structures as it allows 

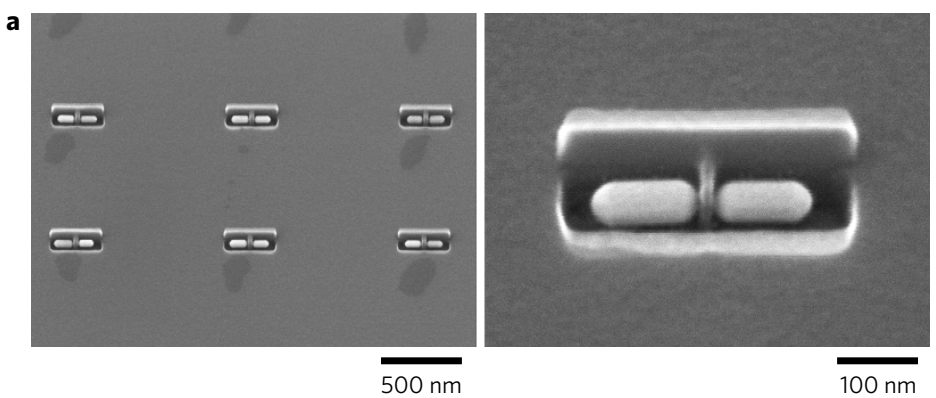
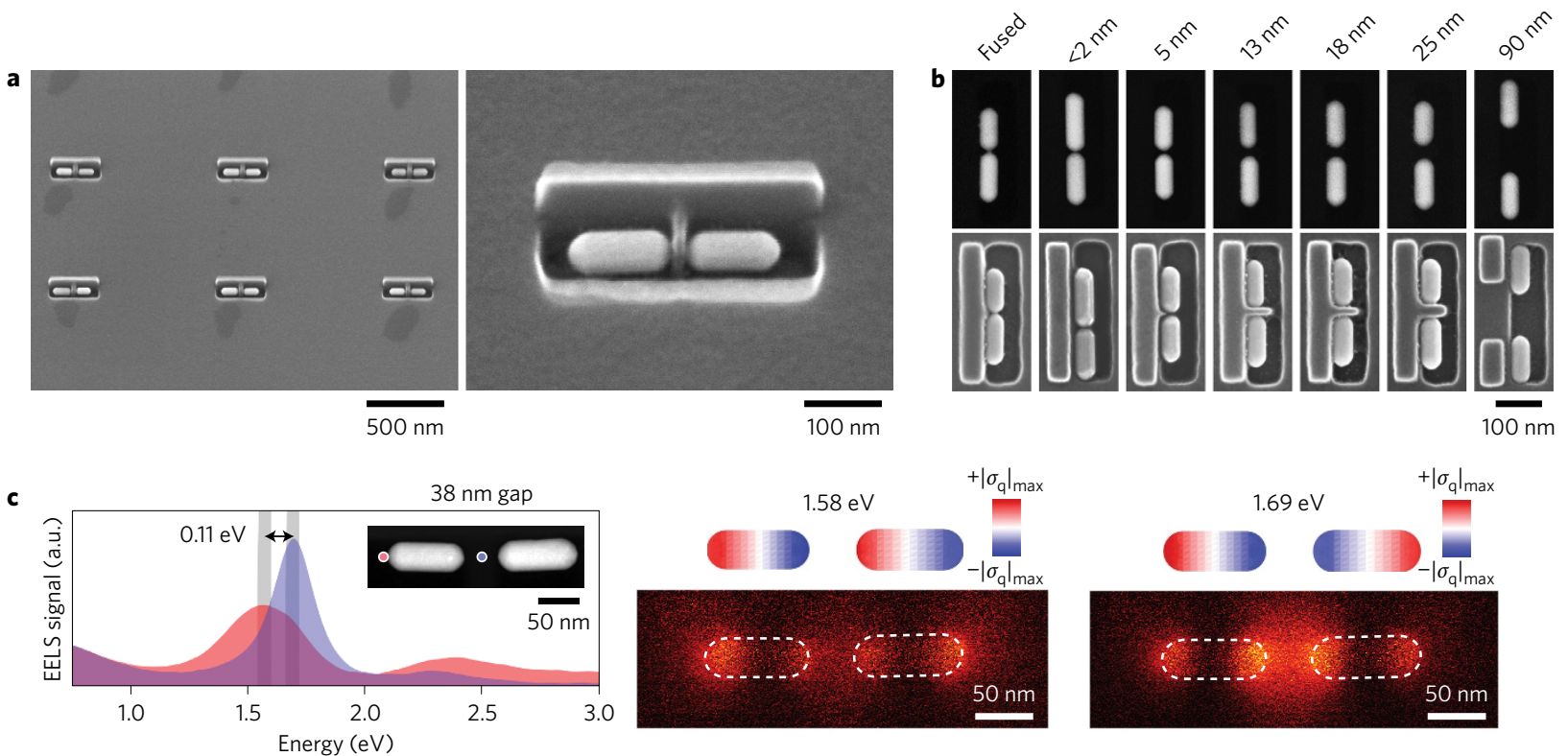

d
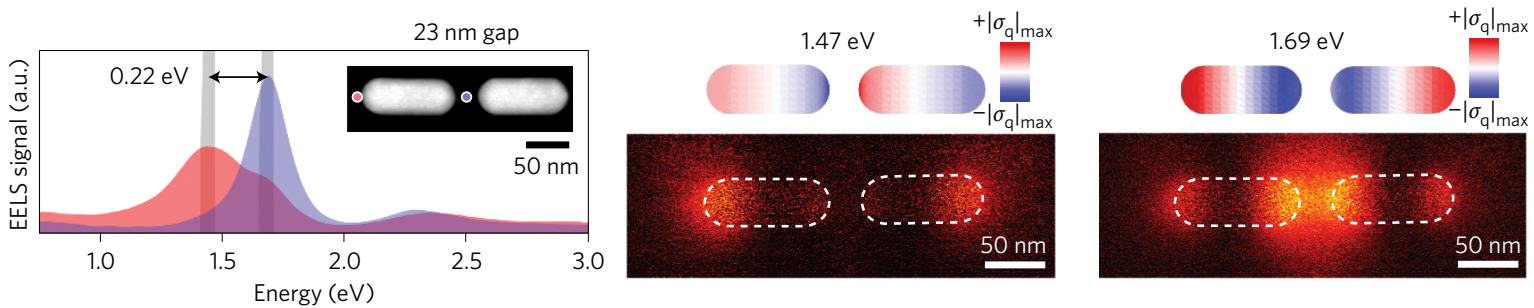

e
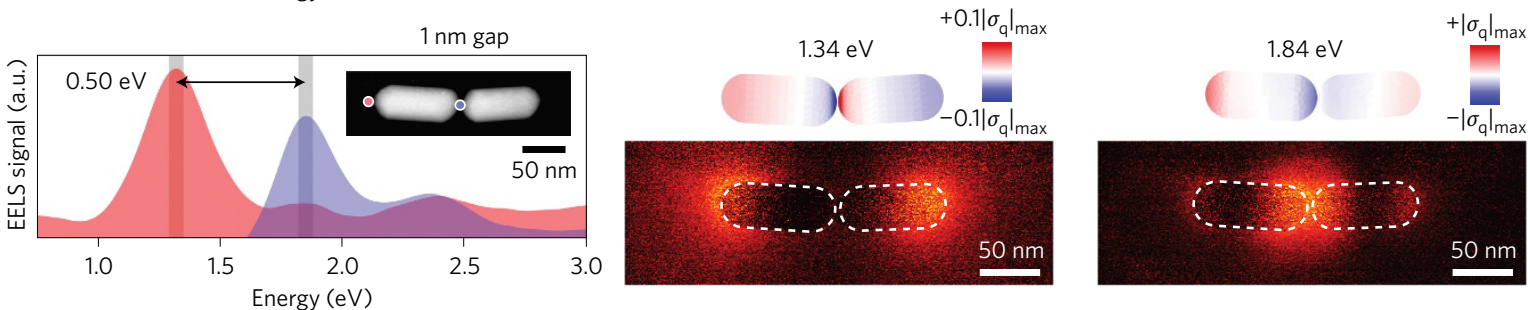

f

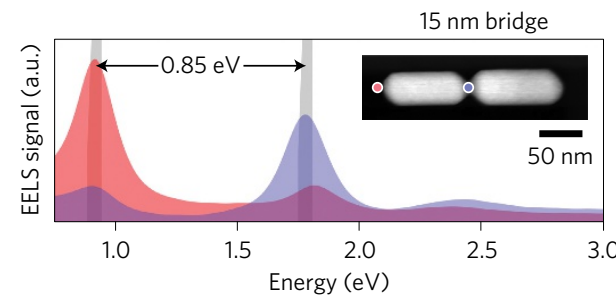

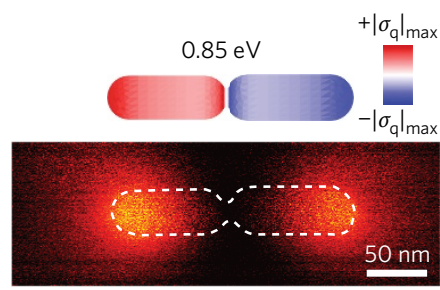

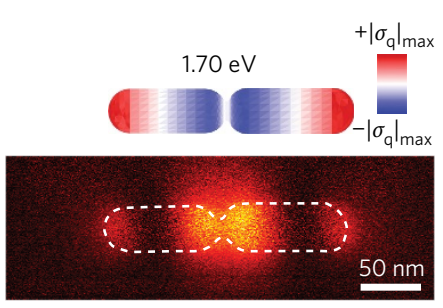

Figure 5 | Spectral response of spatially programmed Au nanorod dimers. a,b, Scanning electron micrographs at $45^{\circ}$ tilt view of Au nanorod dimers assembled within adjacent traps separated by an HSQ spacer (a). The interparticle gap is deterministically controlled by the width of the spacer, as shown in b, where secondary detector images show Au nanorods (top row) and in-lens images show Au nanorods, HSQ spacer and trap edges (bottom row). c-f, Systematic study of the plasmonic mode coupling in nanorod dimers with programmed interparticle gap via scanning transmission electron microscopy electron energy-loss spectroscopy (STEM-EELS). The spectral position of the higher-energy anti-bonding non-radiative mode best sampled in the gap region of the STEM-EELS maps is defined mainly by the nanorod aspect ratio and is relatively insensitive to interparticle distance. The bonding mode, sampled at the dimer side, undergoes strong redshift with reduced gap width. The mode splitting evolves from $0.11 \mathrm{eV}$ in the weakly coupled system at $38 \mathrm{~nm}$ gap (c) to $0.22 \mathrm{eV}$ at $23 \mathrm{~nm}$ gap (d) and ultimately to $0.5 \mathrm{eV}$ for a $1 \mathrm{~nm}$ gap defined by the collapsed CTAB moieties (e). As the nanorod dimer is fused following oxygen plasma treatment and thermal annealing, the dipole mode corresponding to a charge-transfer plasmon redshifts further at $0.85 \mathrm{eV}$ in the near-infrared (f). EELS maps binned over $0.1-0.14 \mathrm{eV}$, as greyed out in the spectrum, and normalized by the intensity of the zero-loss peak. EELS maps and corresponding simulated charge distributions at the observed mode confirm the controlled near-field tuning. $\left|\sigma_{q}\right|_{\max }$ is the maximal charge density for each structure.

for simultaneous morphological imaging and spectral measurements. Aberration-corrected and monochromated electron microscopes have the capability to span a spectral range extending from the ultraviolet to the infrared band while spatially resolving both the electromagnetic resonances of plasmonic nanostructures and imaging antenna geometries at the subnanometre level ${ }^{47,48}$. Tunable plasmonic coupling is evidenced here by the energy splitting of the bonding and anti-bonding longitudinal modes supported by the antennas. For weakly coupled systems, such as the one shown in Fig. $5 \mathrm{c}$ with a gap of $38 \mathrm{~nm}$ between the nanorods, the corresponding bonding and anti-bonding plasmonic modes overlap significantly as they are observed at 1.58 and $1.69 \mathrm{eV}$, respectively. 

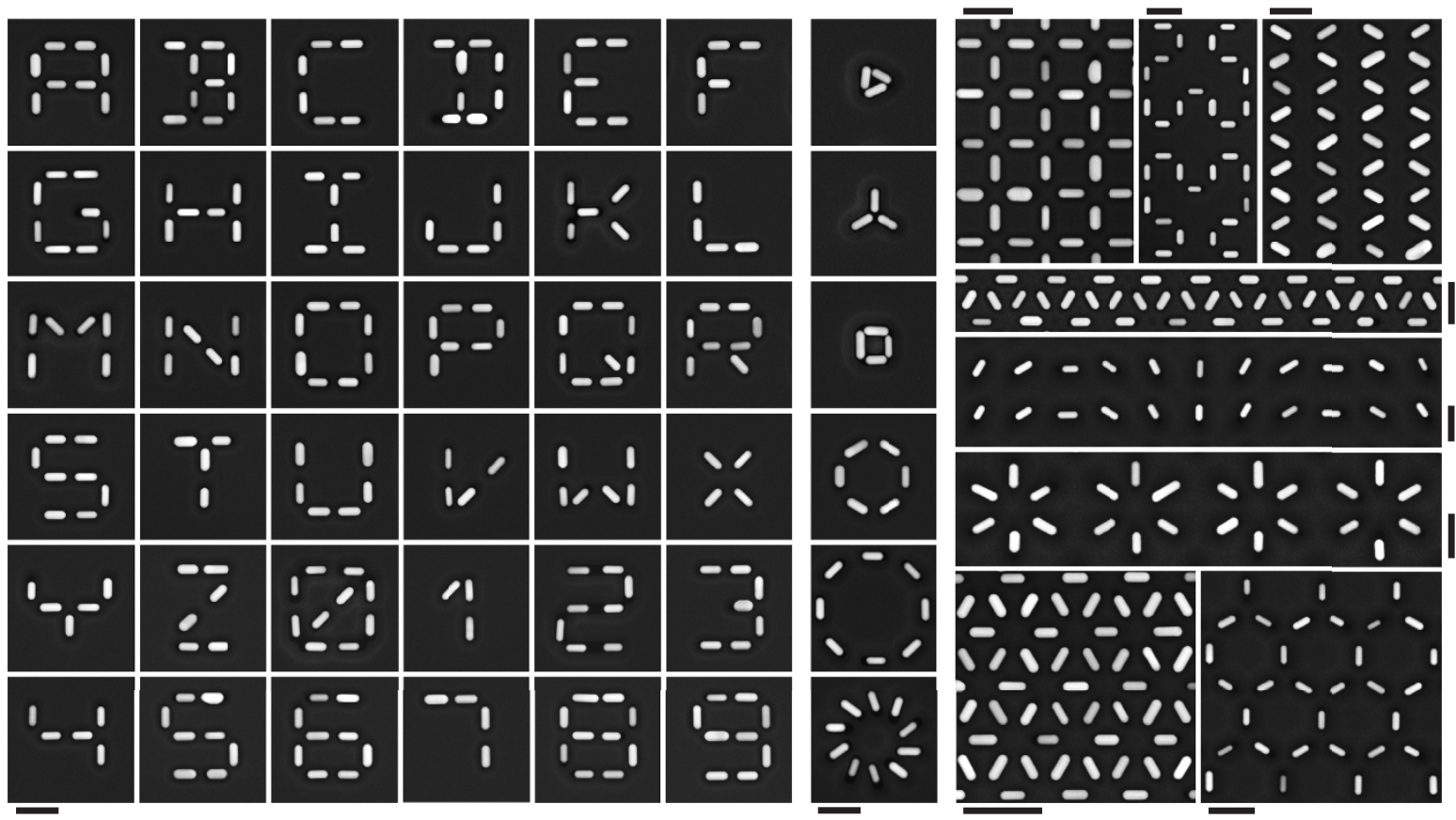

Figure 6 | Examples of two-dimensional patterns of Au nanorods fabricated by topographically templated capillary assembly. This collection of SEM micrographs includes the 26 characters of the Latin alphabet (A-Z) and the Arabic numeral characters (0-9) (left panel), several clusters and geometries (central panel), and lattices of various symmetry and fragments of a Hilbert curve (right panel). These complex motifs were implemented in funnelled traps with arbitrary orientations with respect to the receding contact line. All assemblies were obtained through single runs of nanorod deposition. All scale bars, $250 \mathrm{~nm}$.

Since STEM-EELS is unable to reveal plasmonic modes at locations where the induced electric field is perpendicular to the velocity vector of the impinging electron beam ${ }^{49}$, this method is blind to some of the electromagnetic hotspots resulting from the interactions between the nanoparticles. On the other hand, the plasmonic modes are evidenced by the high energy-loss probability at the edges and centre of the dimers observed in the near-field EELS maps plotted at the respective resonant energies of the bonding and antibonding modes. This behaviour is well observed in the antennas with gaps of $23 \mathrm{~nm}$ (Fig. 5d) and $1 \mathrm{~nm}$ (Fig. 5e), where the energy loss is gradually localized at the expected locations for both modes. Electron energy-loss and scattering simulations based on a surface integral equations method ${ }^{50}$ are in excellent agreement with our experimental data (see Supplementary Information and Supplementary Figs 8 and 9). The simulations confirm the controlled coupling of the plasmonic modes in the dimer nanoantennas and reveal similar gradual energy splitting from $0.11 \mathrm{eV}$ at $38 \mathrm{~nm}$ gap to $0.5 \mathrm{eV}$ in the strong coupling regime (1 nm gap) (Supplementary Fig. 8). By performing geometrically accurate simulations of the charge distributions associated with the nanostructure eigenmodes, we further confirm the physical nature of the observed modes and extract the related near-field distributions (Supplementary Fig. 10). Upon oxygen plasma treatment, the adsorbed collapsed surfactant is removed and dimers initially separated by the surfactant are controllably fused (Fig. 5f). In this bridged dimer case, the anti-bonding mode remains at a similar energy while the bonding mode follows a charge transfer plasmon behaviour and is further redshifted to the short-wavelength infrared ${ }^{42}$. Although weakening the bonding-mode gap enhancement, the narrow bridge enables convenient engineering of antennas resonant at longer infrared wavelengths with compact footprints.

\section{Conclusions}

We have shown how specific topographic patterning of solid substrates can fully determine the capillary assembly of $\mathrm{Au}$ nanorods with $\sim 1 \mathrm{~nm}$ resolution. Such spatial accuracy has been achieved by a detailed understanding of the sequential stages of the capillary assembly dynamics and a systematic analysis of the assembly yields. STEM-EELS studies have confirmed the functionality of the assembled strongly coupled plasmonic antennas. The main limitation of this method for the fabrication of complex multimeric nanostructures currently lies in the polydispersity of the colloidal solution. Finally, we note that our method is largely independent of substrate and nanoparticle composition, as it can be extended to arbitrary surface patterns (Fig. 6) and to out-ofplane orientation as well as to other types of nanoparticle, such as Ag nanocubes (Supplementary Fig. 16). Consequently, capillary assembly of nanoparticles may be competitive with DNA-mediated nanoparticle assembly for the fabrication of plasmonic ${ }^{21}$, nanoelectronic $^{28}$, optoelectronic ${ }^{6}$ and other functional nanodevices ${ }^{29}$ that harness the unique properties of nanoparticle assemblies because of its simplicity, lithographic accuracy and scalability to large substrates.

\section{Methods}

Methods and any associated references are available in the online version of the paper.

Received 18 February 2016; accepted 17 August 2016; published online 3 October 2016

\section{References}

1. Benson, O. Assembly of hybrid photonic architectures from nanophotonic constituents. Nature 480, 193-199 (2011).

2. Fan, J. A. et al. Self-assembled plasmonic nanoparticle clusters. Science 328, 1135-1138 (2010).

3. Law, M., Goldberger, J. \& Yang, P. D. Semiconductor nanowires and nanotubes. Ann. Rev. Mater. Res. 34, 83-122 (2004).

4. Baughman, R. H., Zakhidov, A. A. \& de Heer, W. A. Carbon nanotubes-the route toward applications. Science 297, 787-792 (2002).

5. Mochalin, V. N., Shenderova, O., Ho, D. \& Gogotsi, Y. The properties and applications of nanodiamonds. Nat. Nanotech. 7, 11-23 (2012). 
6. Caruge, J. M., Halpert, J. E., Wood, V., Bulovic, V. \& Bawendi, M. G. Colloidal quantum-dot light-emitting diodes with metal-oxide charge transport layers. Nat. Photon. 2, 247-250 (2008).

7. Goris, B. et al. Plasmon mapping in Au@Ag nanocube assemblies. J. Phys. Chem. C 118, 15356-15362 (2014)

8. Glotzer, S. C. \& Solomon, M. J. Anisotropy of building blocks and their assembly into complex structures. Nat. Mater. 6, 557-562 (2007).

9. Vigderman, L., Khanal, B. P. \& Zubarev, E. R. Functional gold nanorods: synthesis, self-assembly, and sensing applications. Adv. Mater. 24, 4811-4841 (2012).

10. Nepal, D. et al. Control over position, orientation, and spacing of arrays of gold nanorods using chemically nanopatterned surfaces and tailored particle-particle-surface interactions. ACS Nano 6, 5693-5701 (2012).

11. Celebrano, M. et al. Mode matching in multiresonant plasmonic nanoantennas for enhanced second harmonic generation. Nat. Nanotech. 10, 412-417 (2015).

12. Halas, N. J., Lal, S., Chang, W.-S., Link, S. \& Nordlander, P. Plasmons in strongly coupled metallic nanostructures. Chem. Rev. 111, 3913-3961 (2011).

13. Tan, S. J., Campolongo, M. J., Luo, D. \& Cheng, W. Building plasmonic nanostructures with DNA. Nat. Nanotech. 6, 268-276 (2011).

14. Funke, J. J. \& Dietz, H. Placing molecules with Bohr radius resolution using DNA origami. Nat. Nanotech. 11, 47-52 (2016).

15. Acuna, G. P. et al. Fluorescence enhancement at docking sites of DNA-directed self-assembled nanoantennas. Science 338, 506-510 (2012).

16. Schreiber, R. et al. Chiral plasmonic DNA nanostructures with switchable circular dichroism. Nat. Commun. 4, 2948 (2013).

17. Kuzyk, A. et al. Reconfigurable 3D plasmonic metamolecules. Nat. Mater. 13, 862-866 (2014)

18. Lu, W. \& Lieber, C. M. Nanoelectronics from the bottom up. Nat. Mater. 6, 841-850 (2007).

19. Yin, Y., Lu, Y., Gates, B. \& Xia, Y. Template-assisted self-assembly: a practical route to complex aggregates of monodispersed colloids with well-defined sizes, shapes, and structures. J. Am. Chem. Soc. 123, 8718-8729 (2001).

20. Cui, Y. et al. Integration of colloidal nanocrystals into lithographically patterned devices. Nano Lett. 4, 1093-1098 (2004).

21. Henzie, J., Andrews, S. C., Ling, X. Y., Li, Z. \& Yang, P. Oriented assembly of polyhedral plasmonic nanoparticle clusters. Proc. Natl Acad. Sci. USA 110, 6640-6645 (2013).

22. Kraus, T. et al. Nanoparticle printing with single-particle resolution. Nat. Nanotech. 2, 570-576 (2007).

23. Malaquin, L., Kraus, T., Schmid, H., Delamarche, E. \& Wolf, H. Controlled particle placement through convective and capillary assembly. Langmuir 23, 11513-11521 (2007).

24. Mastrangeli, M. The fluid joint: the soft spot of micro- and nanosystems. $A d v$ Mater. 27, 4254-4272 (2015).

25. Greybush, N. J. et al. Plasmon-enhanced upconversion luminescence in single nanophosphor-nanorod heterodimers formed through template-assisted self-assembly. ACS Nano 8, 9482-9491 (2014).

26. Ni, S., Klein, M. J., Spencer, N. D. \& Wolf, H. Cascaded assembly of complex multiparticle patterns. Langmuir 30, 90-95 (2014).

27. Zhou, Y. et al. Shape-selective deposition and assembly of anisotropic nanoparticles. Nano Lett. 14, 2157-2161 (2014).

28. Collet, M. et al. Large-scale assembly of single nanowires through capillary-assisted dielectrophoresis. Adv. Mater. 27, 1268-1273 (2015).

29. Hanske, C. et al. Strongly coupled plasmonic modes on macroscopic areas via template-assisted colloidal self-assembly. Nano Lett. 14, 6863-6871 (2014).

30. Kuemin, C., Nowack, L., Bozano, L., Spencer, N. D. \& Wolf, H. Oriented assembly of gold nanorods on the single-particle level. Adv. Funct. Mater. 22, 702-708 (2012).

31. Ni, S., Leemann, J., Wolf, H. \& Isa, L. Insights into mechanisms of capillary assembly. Faraday Discuss. 181, 225-242 (2015).

32. Kuemin, C., Stutz, R., Spencer, N. D. \& Wolf, H. Precise placement of gold nanorods by capillary assembly. Langmuir 27, 6305-6310 (2011).

33. Frenkel, D. Order through entropy. Nat. Mater. 14, 9-12 (2015).

34. Pinedo Rivera, T. et al. Assisted convective-capillary force assembly of gold colloids in a microfluidic cell: plasmonic properties of deterministic nanostructures. J. Vac. Sci. Technol. B 26, 2513-2519 (2008).
35. Oliver, J. F., Huh, C. \& Mason, S. G. Resistance to spreading of liquids by sharp edges. J. Colloid Interface Sci. 59, 568-581 (1977).

36. Hu, H. \& Larson, R. G. Analysis of the microfluid flow in an evaporating sessile droplet. Langmuir 21, 3963-3971 (2005).

37. Gordon, M. J. \& Peyrade, D. Separation of colloidal nanoparticles using capillary immersion forces. Appl. Phys. Lett. 89, 053112 (2006).

38. Bishop, K. J. M., Wilmer, C. E., Soh, S. \& Grzybowski, B. A. Nanoscale forces and their uses in self-assembly. Small 5, 1600-1630 (2009).

39. Siegfried, T., Ekinci, Y., Martin, O. J. F. \& Sigg, H. Engineering metal adhesion layers that do not deteriorate plasmon resonances. ACS Nano 7, 2751-2757 (2013)

40. Cetin, A. E., Etezadi, D. \& Altug, H. Accessible nearfields by nanoantennas on nanopedestals for ultrasensitive vibrational spectroscopy. Adv. Opt. Mater. 2, 866-872 (2014)

41. Schuller, J. A. et al. Plasmonics for extreme light concentration and manipulation. Nat. Mater. 9, 193-204 (2010).

42. Duan, H. G., Fernandez-Dominguez, A. I., Bosman, M., Maier, S. A. \& Yang, J. K. W. Nanoplasmonics: classical down to the nanometer scale. Nano Lett. 12, 1683-1689 (2012).

43. Scholl, J. A., Garcia-Etxarri, A., Koh, A. L. \& Dionne, J. A. Observation of quantum tunneling between two plasmonic nanoparticles. Nano Lett. 13, 564-569 (2013).

44. Teulle, A. et al. Multimodal plasmonics in fused colloidal networks. Nat. Mater. 14, 87-94 (2015).

45. Jung, H., Cha, H., Lee, D. \& Yoon, S. Bridging the nanogap with light: continuous tuning of plasmon coupling between gold nanoparticles. ACS Nano 9, 12292-12300 (2015).

46. Baffou, G., Girard, C., Dujardin, E., Colas des Francs, G. \& Martin, O. J. F. Molecular quenching and relaxation in a plasmonic tunable system. Phys. Rev. B 77, 121101 (2008).

47. Egerton, R. F. Electron energy-loss spectroscopy in the TEM. Rep. Prog. Phys. 72, 016502 (2009).

48. Nicoletti, O. et al. Three-dimensional imaging of localized surface plasmon resonances of metal nanoparticles. Nature 502, 80-84 (2013).

49. Hörl, A., Trügler, A. \& Hohenester, U. Tomography of particle plasmon fields from electron energy loss spectroscopy. Phys. Rev. Lett. 111, 076801 (2013).

50. Kern, A. M. \& Martin, O. J. F. Surface integral formulation for 3D simulations of plasmonic and high permittivity nanostructures. J. Opt. Soc. Am. A 26, $732-740$ (2009).

51. Deegan, R. D. et al. Capillary flow as the cause of ring stains from dried liquid drops. Nature 389, 827-829 (1997)

\section{Acknowledgements}

The authors thank the staff of the Center of Micro/Nanotechnology (CMI) of EPFL for the valuable discussions and support. This research was funded by the European Commission (FP7-ICT-2011-7, NANO-VISTA, under grant agreement no. 288263), the Interuniversity Attraction Poles program MicroMAST (IAP 7/38) initiated by the Belgian Science Policy Office, and the Swiss National Science Foundation project 200020_153662.

\section{Author contributions}

V.F. conceived the research, designed the experiments, built the experimental set-up, fabricated the substrates, performed the assembly experiments, analysed assembly and STEM-EELS data, and wrote the manuscript. M.M. conceived the research, designed the assembly experiments, analysed the assembly data and wrote the manuscript. G.D.B. and J.B. performed the numerical simulations and revised the manuscript. D.T.L.A. performed the STEM-EELS measurements, analysed the corresponding data and revised the manuscript. E.S. performed the assembly experiments and analysed the assembly data. O.J. F.M. supervised the numerical simulations and revised the manuscript. J.Br. supervised the research and wrote the manuscript.

\section{Additional information}

Supplementary information is available in the online version of the paper. Reprints and permissions information is available online at www.nature.com/reprints. Correspondence and requests for materials should be addressed to J.B.

\section{Competing financial interests}

The authors declare no competing financial interests. 
Methods

Nanoparticle preparation. Suspensions of nominally $110 \mathrm{~nm} \times 40 \mathrm{~nm} \times 40 \mathrm{~nm} \mathrm{Au}$ nanorods stabilized with an adsorbed monolayer of cetyl trimethylammonium bromide (CTAB) were purchased from Nanopartz (USA) at a concentration of $2.64 \times 10^{10} \mathrm{ml}^{-1} .500 \mu \mathrm{l}$ of the as-purchased nanorod suspension were centrifuged twice for $10 \mathrm{~min}$ at $5,000 \mathrm{rpm}$ in $2 \mathrm{ml}$ of CTAB solution at its critical micelle concentration (CMC, $0.9 \mathrm{mM}$ ) to increase the concentration of the nanorods (up to $2.54 \times 10^{10} \mathrm{ml}^{-1}$ ) and control the supernatant properties. Fifty microlitres of the resulting concentrated solution was sonicated for $5 \mathrm{~min}$ and used in assembly experiments immediately after preparation. The thickness of the adsorbed CTAB monolayer $(3 \mathrm{~nm})$ extends the effective interaction radius of the suspended nanorods beyond their nominal diameter. The CTAB monolayers still allow for gaps narrower than $2 \mathrm{~nm}$ between adjacent nanorods in the final structures due to collapse upon drying. Post-assembly measurements of the size distribution of $\mathrm{Au}$ nanorods from SEM imaging are shown in Supplementary Fig. 1.

The Ag nanocubes shown in Supplementary Fig. 15 had nominal edge length of $100 \mathrm{~nm}$ (Nanocomposix, Czech Republic) and were prepared following a similar procedure as for the nanorods.

Template fabrication. The topographical templates were fabricated on silicon wafers (100 mm diameter, prime grade) and silicon nitride membranes (low-stress low-pressure chemical vapour deposition) by means of multiple aligned steps of electron-beam lithography (VISTEC EBPG5000+, $100 \mathrm{kV}$ ) and dry etching. This allowed the capillary assembly of the Au nanorods to be performed under comparable conditions on traps with different geometric profiles, orientations, depths and pitch simultaneously. The trap types were organized into parallel arrays periodically repeated along the direction swept by the colloidal meniscus, spanning a $5 \times 5 \mathrm{~mm}^{2}$ surface of the $17 \times 17 \mathrm{~mm}^{2}$ substrates. After completion of the process, 4,800 traps of each trap type with varying width and orientation were analysed to extract statistics on assembly yield and positioning accuracy.

All apertures were defined using ZEP-520A resist developed at room temperature for $60 \mathrm{~s}$ in $n$-amyl acetate and rinsed in methyl isobutyl ketone/isopropyl alcohol (9:1) for $60 \mathrm{~s}$ before being blow dried with a nitrogen gun. The samples were subsequently dry etched with $\mathrm{Cl}_{2}$ - (STS Multiplex ICP) or $\mathrm{C}_{4} \mathrm{~F}_{6}$-based chemistries (Alcatel 601E) to pattern silicon and silicon nitride substrates, respectively. The funnel profile was obtained by exploiting resist faceting during $\mathrm{Cl}_{2}$-based inductively coupled plasma reactive ion etching of silicon. Using 150-nm-thick layers of resist (ZEP 520-A 50\% in anisole, 2,500 rpm), the faceted resist profile was transferred to the underlying substrate. All auxiliary sidewalls were patterned in hydrogen silsesquioxane (HSQ) (XR-1541-002 and XR-1541-004) with thickness between 30 and $40 \mathrm{~nm}$ and aligned to the previously etched layer with accuracies better than $10 \mathrm{~nm}$. The HSQ was either used as functional material or transferred to the underlying layer by dry etching. The substrates then were mechanically diced, surface-activated by exposure to oxygen plasma (Tepla Gigabatch, 1,000 W, $5 \mathrm{~min}, 500$ s.c.c.m. $\mathrm{O}_{2}$, where s.c.c.m. is standard cubic centimetres per minute), and finally rendered hydrophobic by vapour-phase absorption of $1 \mathrm{H}, 1 \mathrm{H}, 2 \mathrm{H}, 2 \mathrm{H}$-perfluorodecyltrichlorosilane (Aldrich) under vacuum for $1 \mathrm{~h}$. The wettability of the substrates was characterized through static contact angle measurements by the sessile drop method before experiments, obtaining values in excess of $110^{\circ}$ for water and $63^{\circ}$ for CTAB solution at its CMC at room temperature.

The low-stress, silicon-rich nitride membranes used for the STEM-EELS study were processed following similar procedures and further released by backside dry etching (AMS200 Bosch process) and wet etching (KOH 40\%).

Nanoparticle assembly. The assembly process was carried out under an upright microscope (Zeiss Axio Scope A1) using a home-built set-up. The set-up controlled the motion of the topographical template relative to a transparent glass coverslip, which was held fixed on top of the template. A controlled volume of nanoparticle solution $(50-100 \mu \mathrm{l})$ was injected between the template and the glass coverslip separated by a vertical gap of $500 \mu \mathrm{m}$. The glass coverslip was rendered hydrophobic using the same silanization procedure used to chemically condition the substrate, described in the previous section. A Peltier element was used to set the temperature of the substrate to control the rate of solvent evaporation and the accumulation of nanoparticles at the receding contact line of the meniscus. The Peltier element was combined with a vacuum stage and placed under the substrate. The substrate temperature was typically set $30^{\circ} \mathrm{C}$ above the dew point and regulated by means of a proportional-integral-derivative controller. The substrate was set in motion through a motorized axis (PI Micos, PLS-85). The process was monitored optically in real time with the upright optical microscope under dark-field and cross-polarized mode, along with a side-view camera recording the dynamic receding contact angle of the confined meniscus on the template. The receding contact angle measured $80^{\circ}$ at the beginning of the experiments and reached $50^{\circ}$ when steady-state nanoparticle accumulation was reached.
The sliding speed was tailored to form a sufficiently dense accumulation zone, avoid unselective nanoparticles unloading onto the substrate, and minimize processing duration. A too high sliding speed can generate a strong recirculation flow within the colloidal meniscus that may ultimately hinder dense nanoparticle accumulation in proximity of the receding contact line. A speed of $\sim 2 \mu \mathrm{m} \mathrm{s}^{-1}$ provided the best assembly results onto sparse arrays of monomeric and dimeric traps. Dense and more articulated trap arrays induce more pronounced pinningunpinning dynamics of the receding contact line, and in this case a speed between 1 and $1.4 \mu \mathrm{m} \mathrm{s}^{-1}$ provided the highest assembly yields. The capillary assembly of nanoparticles was in all instances preceded by a conditioning phase, whereby the colloidal meniscus is dragged across a non-patterned area of the pre-heated substrate for up to $20 \mathrm{~min}$ to achieve steady evaporative and flow conditions and stable nanoparticle accumulation prior to crossing over the nanopatterned traps. A full assembly run was typically completed in about $1 \mathrm{~h}$.

Assembly yield and angular analysis. We define the assembly yield as the ratio of the number of traps filled with at least one nanorod and the total number of traps (designed to host a single nanorod) in the given array. Non-selective deposition of the nanords outside the traps was observed in extremely rare cases; these events were checked and not accounted for in the total yield. It should additionally be noted that straight-edged traps of 60 or $70 \mathrm{~nm}$ width and depth of $100 \mathrm{~nm}$, as well as funnelled traps of similar width, exhibited assembly yield close to $100 \%$, but this result includes in some cases the capture of multiple nanorods into the single traps, which are therefore not of interest for our work targeting fully controlled structures.

During the capillary assembly experiments, the receding contact line of the colloidal meniscus crossed simultaneously groups of arrays of traps spanning a wide region of the parameter space. The traps differed in relative orientation $\left(0^{\circ}, 45^{\circ}, 90^{\circ}\right)$ with respect to the direction of the receding contact line, width (from 30 to $70 \mathrm{~nm}$ in steps of $10 \mathrm{~nm}$ ), depth (28 or $100 \mathrm{~nm}$ ), funnelled versus straight-edged profile, and decoration with single HSQ auxiliary sidewall (either no HSQ sidewall, sidewall on the trailing edge or on the pinning edge). A total of 135 arrays, each containing 1,600 traps, composed a single group of traps, and the groups were iterated along lines parallel to the direction of meniscus motion, cumulatively covering an area of $5 \times 5 \mathrm{~mm}^{2}$. Several of these lines were analysed after CAN and, for each combination of trap features, the full statistics on assembly data were extracted from image analysis of at least 3 groups of 135 arrays ( $>640,000$ traps) that were crossed by the accumulation zone after its steady state was enforced.

In the corresponding plots (Figs 2-4), data points represent mean values and error bars correspond to the standard deviation of the assembly yield. Interquartile range was used to quantify the dispersion in the orientation relative to the main trap axis of the assembled nanorods, given the observed skewedness of the corresponding angular distributions. Images for the analysis of nanorods' assembly yield and angular orientation were acquired by a scanning electron microscope (Zeiss Merlin) at $20 \mathrm{kV}$ and $5 \mathrm{nA}$ probe current using the secondary electron detector to provide material contrast sufficient to distinguish the Au nanorods from the substrate and trap topography. Assembly yield images were recorded with a resolution of $4 \mathrm{~nm}$ per pixel and angular distribution images at $1 \mathrm{~nm}$ per pixel. ImageJ software and a custom Matlab toolbox were used for image post-processing. The bottom monolayers of the accumulation zone (Supplementary Figs 2 and 3) were imaged by SEM at $1 \mathrm{kV}, 70 \mathrm{pA}$ and analysed following a similar procedure. The stripping from the template of the accumulation zone produced during CAN (Supplementary Fig. 3) was carried out after complete drying of the nanorods by casting a drop of ultraviolet (UV) curable polymer (OrmoComp Microresist GMBH) between the substrate and a glass coverslip. After UV curing, the coverslip was separated from the assembly template revealing the nanorod layers previously in direct contact with the surface of the template.

EELS measurements. STEM-EELS maps were acquired using a FEI Titan Themis 60-300 equipped with a Wien-type monochromator and a Gatan GIF Quantum ERS spectrometer. A $300 \mathrm{keV}$ incident electron beam was used for all experiments, monochromated to give an energy spread of $\sim 110 \mathrm{meV}$ full width at half maximum in the zero-loss peak of elastically scattered electrons, and with beam currents of $\sim 150-230 \mathrm{pA}$. A $17 \mathrm{mrad}$ convergence semi-angle of the probe and a $22 \mathrm{mrad}$ collection semi-angle on the spectrometer were used, with the probe having a mean diameter of $<1 \mathrm{~nm}$ for full width at tenth of maximum in incident intensity. Mapping was performed using the 'ultrafast' spectrum imaging mode with typical dwell times of $0.25-0.5 \mathrm{~ms}$ per pixel, and with the probe rastered in $X, Y$ step sizes of $0.5-0.6 \mathrm{~nm}$ for a total of $>10^{5}$ pixels per map. Each map was treated with the HQ Dark Correction plugin to reduce noise associated with dark current subtraction. Using this approach, the resultant plasmon maps have excellent spatial statistics when considering the inherently delocalized nature of this type of excitation. 